\title{
Case Report: Hepatic Artery Infusion Chemotherapy After Stage I ALPPS in a Patient With Huge HCC
}

\author{
Wenfeng Zhuo, Ang Li, Weibang Yang, Jinxin Duan, Jun Min* and Jinxing Wei* \\ Department of Hepatobiliary Surgery, Sun Yat-sen Memorial Hospital, Sun Yat-sen University, Guangzhou, China
}

Associating liver partition and portal vein ligation for staged hepatectomy (ALPPS) can induce rapid hypertrophy of the liver remnant. However, with a background of liver cirrhosis or other chronic liver diseases, patients with a huge hepatocellular carcinoma (HCC) may sometimes face insufficiency of hepatocellular regeneration after associating liver partition and portal vein ligation for staged hepatectomy (ALPPS). Herein, we report a 56 -year-old male with a vast HCC $(13.3 \times 8.5 \times 13 \mathrm{~cm})$ whose ratio of the future liver remnant (FLR)/standard liver volume (SLV) was $28.7 \%$ when the disease was first diagnosed. Inadequate hypertrophy of FLR was shown in postoperative volumetric assessment a month after stage I ALPPS. After multidisciplinary team discussion

OPEN ACCESS

Edited by:

Marcos Vinicius Perini,

The University of Melbourne, Australia

Reviewed by:

Ivan Romic,

University Hospital Centre

Zagreb, Croatia

Alessandro Coppola,

Policlinico Universitario Campus

Bio-Medico, Italy

${ }^{*}$ Correspondence:

Jun Min

drminj@qq.com

Jinxing We

wjx_870530@163.com

Specialty section:

This article was submitted to

Visceral Surgery,

a section of the journal

Frontiers in Surgery

Received: 24 July 2021

Accepted: 27 October 2021

Published: 26 November 2021

Citation:

Zhuo W, Li A, Yang W, Duan J, Min J and Wei J (2021) Case Report: Hepatic Artery Infusion Chemotherapy After Stage I ALPPS in a Patient With

Huge HCC. Front. Surg. 8:746618.

doi: 10.3389/fsurg.2021.746618
(MDT), the patient was decided to follow three courses of hepatic arterial infusion chemotherapy (HAIC) with oxaliplatin, fluorouracil, and leucovorin (FOLFOX4). The last HAIC was performed together with transhepatic arterial embolization (TAE). Finally, ratio of the FLR/SLV increased from $28.7 \%$ to $40 \%$ during three-month intervals, meeting the requirements of the surgery. Stage II ALPPS, right trisectionectomy, was then successfully performed. There was no recurrence at half years of follow-up. In our case, HAIC seems to be more potent than transcatheter arterial chemoembolization (TACE) in maintaining the hyperplasia of the liver remnant, reducing tumor load, and preventing tumor progression in patients with a large HCC during ALPPS procedure. HAIC, following the first step of ALPPS, a pioneering treatment modality aiming for inadequate hypertrophy of FLR induced by ALPPS, could be an alternative procedure for patients with a vast $\mathrm{HCC}$ in clinical practice.

Keywords: ALPPS, hepatocellular carcinoma, HAIC, FLR, hypertrophy, case report

\section{BACKGROUND}

Hepatocellular carcinoma (HCC), with high incidence and mortality worldwide, has a very poor prognosis. Studies have shown that, except for liver transplantation, surgical resection is the most effective therapy for patients with HCC. However, most patients have lost the opportunity of radical resection owing to the intermediate and advanced stage of the disease when diagnosed (1). ALPPS, a novel surgical approach proposed for the deficiency of the FLR after hepatectomy, is currently mainly suitable for patients with colorectal cancer liver metastasis or with a huge HCC $(>10 \mathrm{~cm})(2)$.

By inducing rapid hypertrophy of the FLR, ALPPS can strive for the chance of radical resection for patients with HCC who have lost it. However, most patients with HCC have a background of liver cirrhosis. Thus, the hypertrophy of the FLR after stage I ALPPS may be inadequate, and stage II ALPPS cannot be carried out (3). 

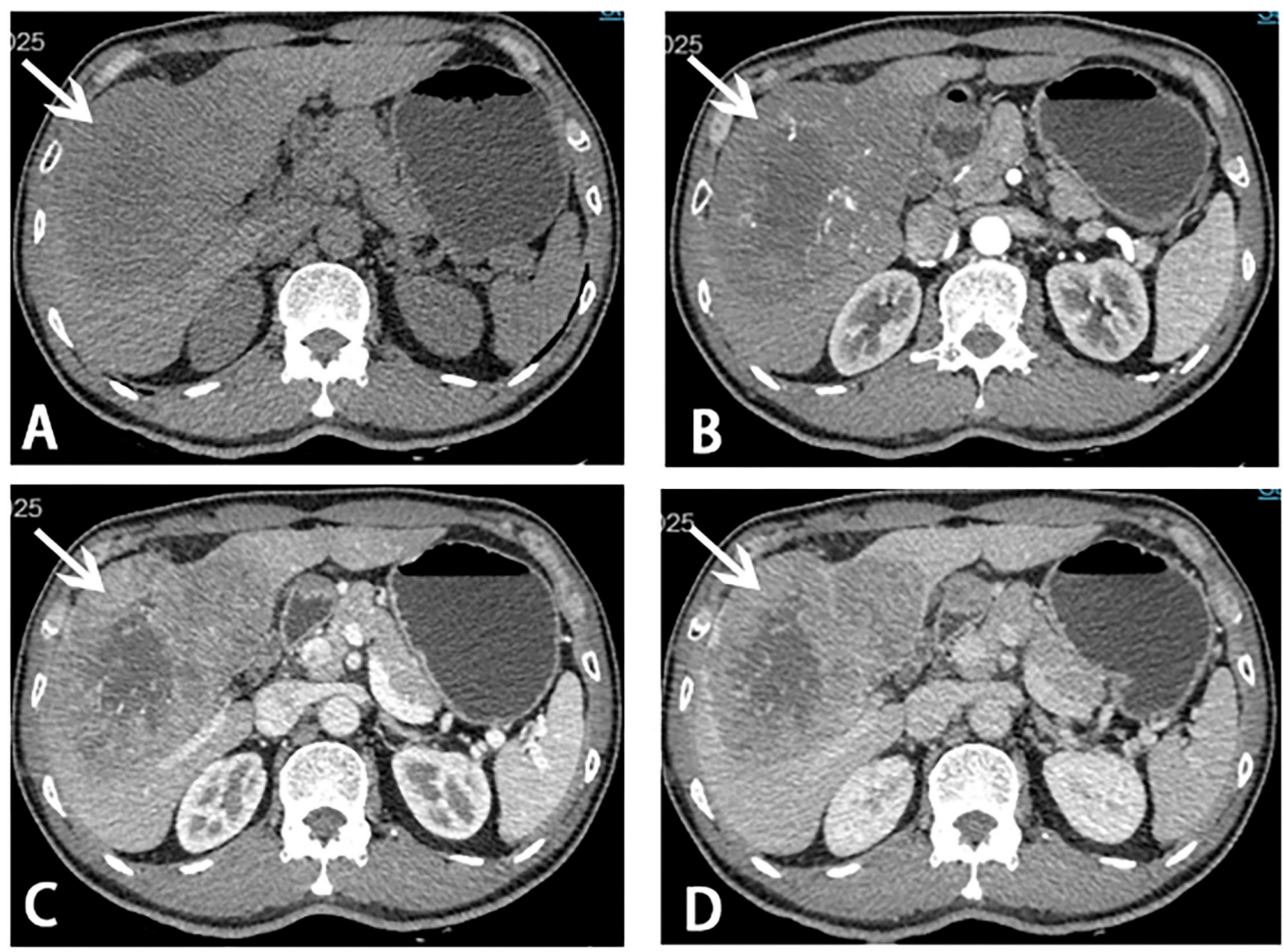

FIGURE 1 | Computed tomography (CT) scans before stage I ALPPS. A giant mass (white arrow) measuring $13.3 \times 8.5 \times 13.0 \mathrm{~cm}$ in liver segments $4,5,6,7$, and 8 was showed on CT scans. (A) Plain scan; (B) arterial phase; (C) portal venous phase; (D) venous phase.

Here, we reported a patient with a huge HCC treated in our center successfully. With insufficient hypertrophy of the FLR after stage I ALPPS, the patient was decided to follow the treatment of HAIC. The FLR then smoothly increased and finally met the requirements of surgery, so that stage II ALPPS, right trisectionectomy, can be successfully performed.

\section{CASE PRESENTATION}

A 56-year-old male with the absence of clinical symptoms was diagnosed as having HCC with a huge tumor $(13.3 \times 8.5 \times$ $13 \mathrm{~cm}$ ) in liver segments $4,5,6,7$, and 8 (Figures 1A-D). The patient has a history of hepatitis B with mild cirrhosis but without any co-morbidities such as hypertension or diabetes. Laboratory tests indicated that liver function, blood routine examination, prothrombin time, and international normalized ratio (INR) were within the normal range. The alpha-fetoprotein (AFP) level was $231 \mathrm{ng} / \mathrm{ml}$ (normal range: $0-7 \mathrm{ng} / \mathrm{ml}$ ), and the Child-Pugh score was 5 . The indocyanine green retention rate at $15 \mathrm{~min}$ (ICG
R15) was $2.7 \%$. There was no evidence of extrahepatic metastases in a CT scan. Therefore, the patient was staged as BCLC-B according to the staging system of the Barcelona Clinic Liver Cancer (BCLC). The estimated preoperative SLV was $1,216 \mathrm{ml}$, and the FLR was $350 \mathrm{ml}$ evaluated with a three-dimensional CT reconstruction system. The FLR/SLV was $28.7 \%$, far $<40 \%$. There would be a high rate of post hepatectomy liver failure (PHLF) if radical liver resection was directly carried out (4). After an MDT, ALPPS was considered as the optimal choice to handle such a situation, and we followed it.

\section{Stage I ALPPS}

The surgery was performed using a reverse "L" incision. Initially, the gallbladder was routinely removed. After anatomical separation, the right portal vein was identified and clipped with a hemo-lock. Liver parenchymal transection was performed with anterior approaches using an ultrasonic scalpel along the transection plane $1 \mathrm{~cm}$ right to the falciform ligament. During this procedure, the hepatic pedicle of liver segment 4 was 

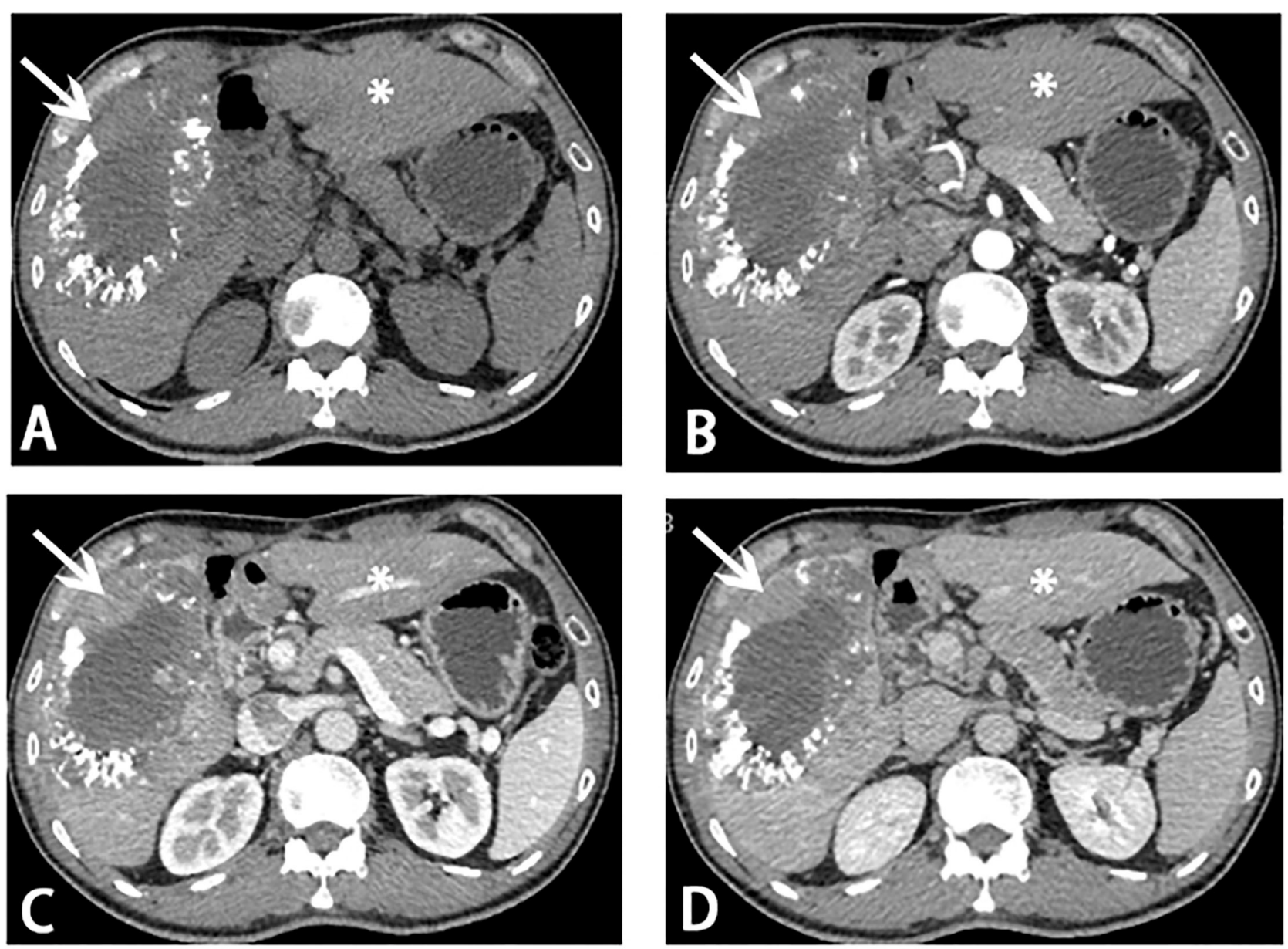

FIGURE 2 | Computed tomography (CT) scans before stage II ALPPS. Reduce of tumor load (white arrow) and hypertrophy of segments 2 and 3 (asterisk) was showed on CT scans. (A) Plain scan; (B) arterial phase; (C) portal venous phase; (D) venous phase.

identified along the right side of the sagittal part of the portal vein and was cut off. The liver was eventually cut into two parts along the above resection plane, while the liver parenchymal close to the first hepatic hilum and in front of the inferior vena cava has remained. The right hepatic pedicle could be easily observed at this time, and we signed it with dark red tape. Finally, the resection plane was covered with an absorbable gelatin sponge, and a surgical drain was routinely placed on the cut surface of liver parenchyma. The duration of surgery was $6 \mathrm{~h}$, and the estimated blood loss was $150 \mathrm{ml}$. A month later, re-evaluation of FLR/SLV by CT three-dimensional reconstruction was 32\%, still, $<40 \%$, which was not suitable for stage II ALPPS (4). Further interventions were needed to be carried out.

\section{HAIC and TAE}

After the MDT, a new method, HAIC with FOLFOX4, was decided to be used for this patient. The patient was given three courses of HAIC therapy with a modified FOLFOX4 regimen (oxaliplatin $150 \mathrm{mg}$ infusion for $2 \mathrm{~h}$ on day 1 , leucovorin 200 $\mathrm{mg} / \mathrm{m}^{2}$ infusion for $2 \mathrm{~h}$ on days 1 and 2 , and fluorouracil 400 $\mathrm{mg} / \mathrm{m}^{2}$ in bolus within $10 \mathrm{~min}$ on days 1 and 2 , and then $2,000 \mathrm{mg}$ continuous infusion for $48 \mathrm{~h}$ ) on August 27, September 24, and November 05, 2020, respectively. The last HAIC was performed together with TAE (two arterial branches supplying the tumor were selected to completely block). After receiving about 3 months of local therapy, the follow-up imaging (Figures 2A-D) showed that the tumor load was reduced, and the FLR/SLR reached $40 \%$ (Figures 3A,B), which meets the requirements of the surgery.

\section{Stage II ALPPS}

Before the operation, the ICG R15 was $4.7 \%$, and the AFP was $8.06 \mathrm{ng} / \mathrm{ml}$. At initial surgical exploration, hypertrophy of the left lateral segment could be easily observed (Figure 3C). The right hepatic pedicle was cut off using a linear cut stapler (LCS) after adhesion separation. Then, the remaining liver parenchyma close to the first hepatic hilum and in front of the inferior vena cava was continuously separated with an ultrasonic scalpel (Figure 3D). The right hepatic vein (RHV) and inferior right posterior vein (IRHV) were both cut off 

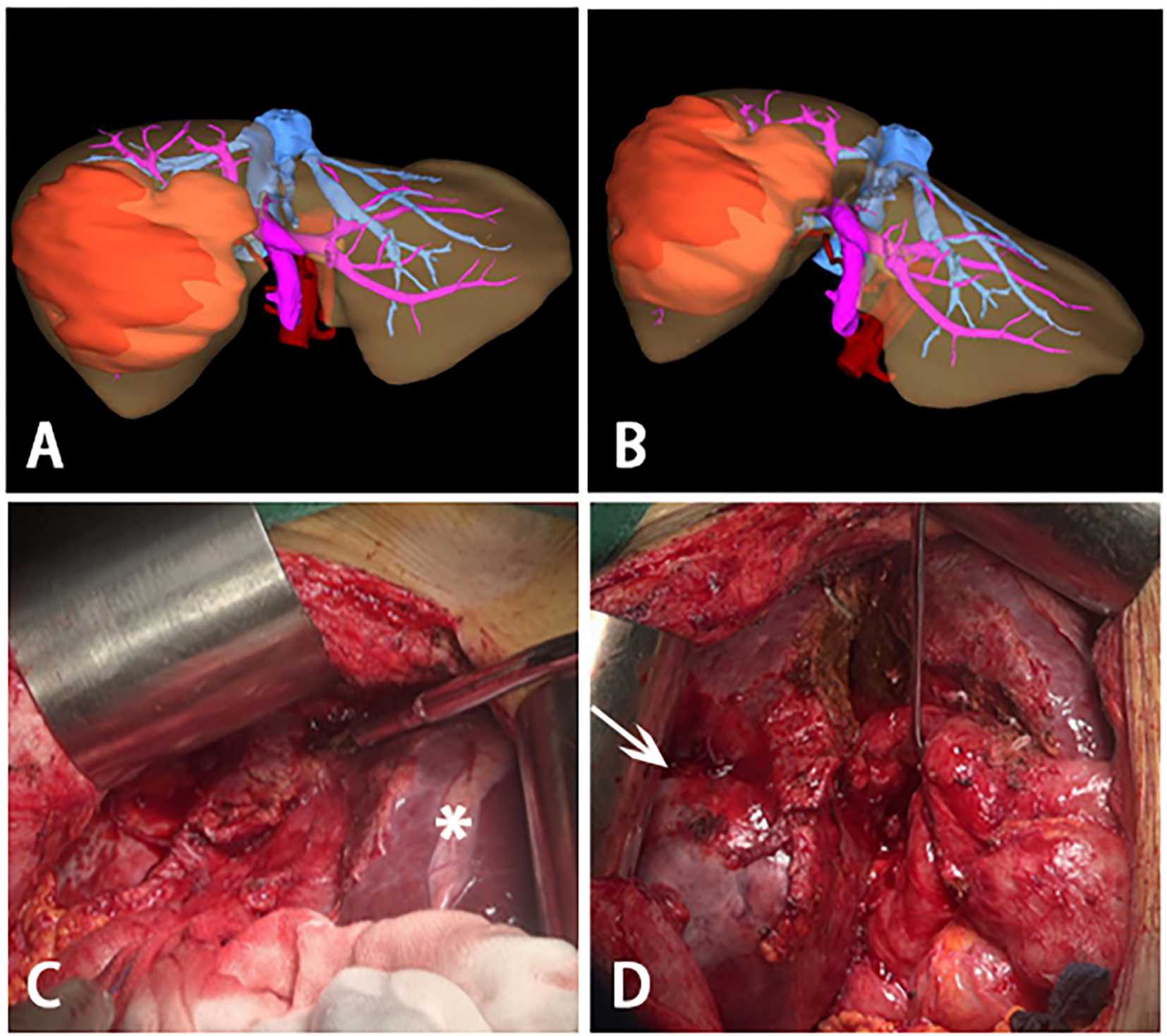

FIGURE 3 | Three-dimensional reconstructions before stage I| ALPPS and intraoperative photographs during stage II ALPPS. (A,B) Three-dimensional reconstructions before stage II ALPPS, a large tumor was on the right lobe of liver and most of the liver parenchymal between right and left was divided. (C) hypertrophy of the left lateral segment (asterisk). (D) process to remove the huge tumor (white arrow)- right trisectionectomy.

with the LCS. The right trisectionectomy was carried out until the diseased liver was totally removed. Lastly, an absorbable gelatin sponge and a surgical drain were placed over the transection plane. The operating time was $310 \mathrm{~min}$, and blood loss was about $500 \mathrm{ml}$. Two units of packed red blood cells were transfused the night right after the surgery, and no eventful complications occurred during postoperative recovery. The histopathology of the tumor confirmed a $12.5 \times 9 \times 8.2 \mathrm{~cm}$ HCC in liver segments $4,5,6,7$, and 8 . The pathology report of this tumor was moderately and poorly differentiated HCC, T1N0M0, stage IB (AJCC Cancer Staging Manual, 2018) (Figures 4A-D). There was no recurrence at half years of follow-up.

\section{DISCUSSION}

Hepatocellular carcinoma is one of the most common malignancies worldwide. According to studies, liver transplantation and radical hepatic resection are the only curative treatments for patients with HCC. The development of liver transplantation was severely limited because of a chronic shortage of viable donor organs (5). Hence, surgical resection is defined as the most promising treatment to improve the prognosis of patients with HCC. For patients without cirrhosis, FLR/SLR $\geq 30 \%$, or for patients with cirrhosis, FLR/SLR $\geq 40 \%$, if the evaluation of liver reserve function (ICG R15) is within a reasonable range, radical hepatectomy is allowed to be carried 

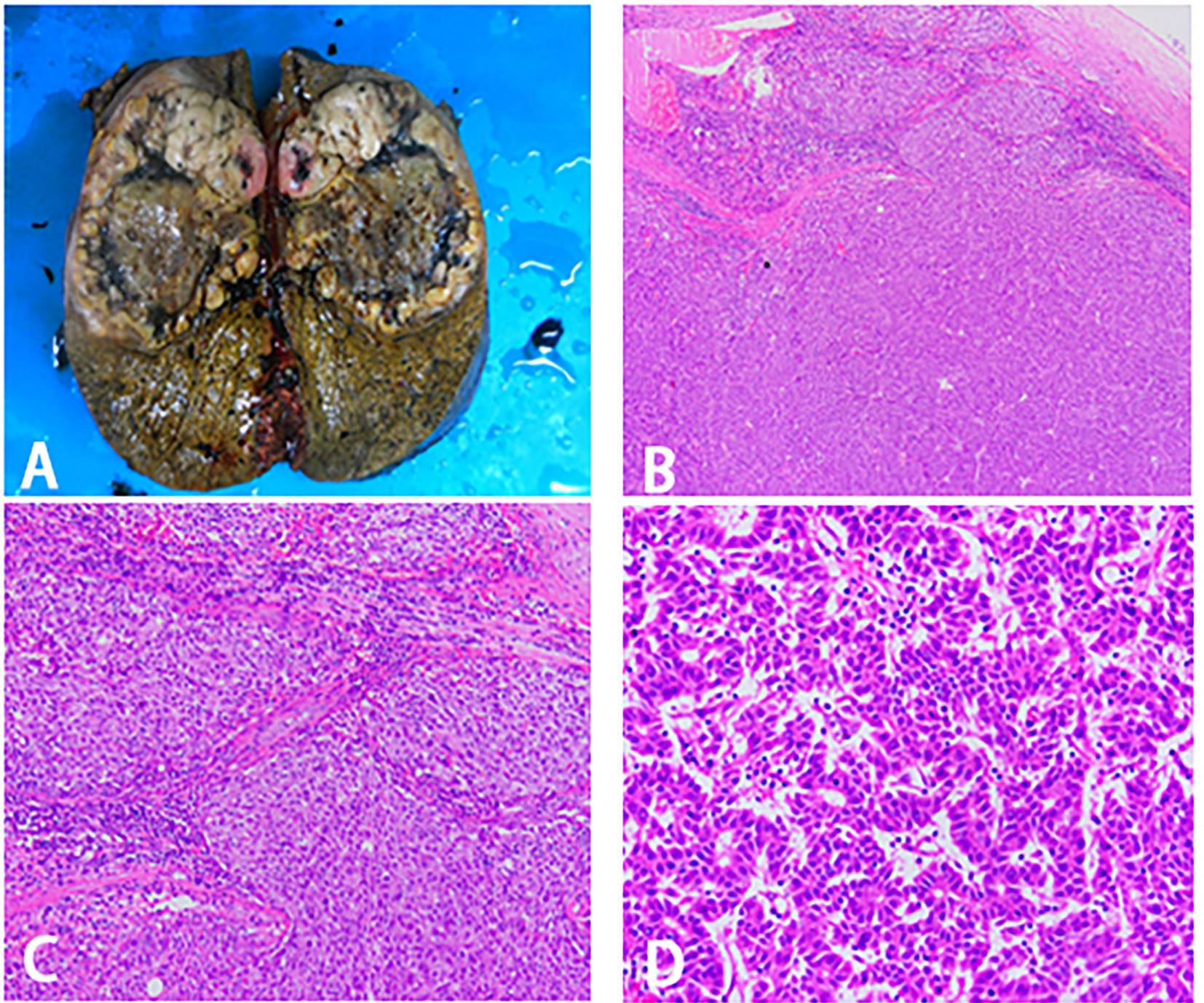

FIGURE 4 | Surgical specimen and histopathological images. (A) Right trisectionectomy specimen. (B-D) Pathological examination of tissue section. (B) 40X magnification. (C) 100X magnification. (D) 400X magnification.

out (6). However, only $30 \%$ of patients with HCC are amenable to surgery when the disease is first diagnosed (1).

Reviewing this case, we can find that it had distinguishing characteristics as follows: 1. The size of HCC was enormous $(>10 \mathrm{~cm})$ when the patient was first diagnosed. 2 . The patient had a background of cirrhosis. 3. FLR/SLR was far less than $40 \%$. A high probability of PHLF would happen if radical hepatectomy was performed directly. Nowadays, for patients with a large HCC who intend to perform surgery, the mainstream modality is to conduct portal vein embolization (PVE) preoperatively to acquire adequate hypertrophy of the FLR (7). Nevertheless, it takes a long time for the hypertrophy of FLR to occur (6-8 weeks), and its success rates are not high. During this relatively long waiting time, there is a high probability of tumor progression; finally, the opportunity of radical surgery is lost (8). Although B. Romic has once pointed out a new method to treat patients with huge HCC by TACE combined with ALPPS, there is weak evidence to support it (9). Thus, given the good general health of the patient, good liver function, mild cirrhosis, and rich clinical experience of ALPPS in our center, ALPPS, which can acquire rapid hypertrophy of FLR, was selected to strive for a higher chance of curative surgery (10). Unfortunately, despite the successful completion of stage I ALPPS, postoperative evaluation still suggested insufficient FLR, as the FLR/SLV was $32 \%$, still $<40 \%$. This low speed of hyperplasia may be related to the background of chronic liver disease (3).

A rare case has reported that after the failure of stage I ALPPS, salvage transhepatic arterial embolization (TAE) could gain the opportunity of radical hepatectomy for patients with a huge HCC (11). Considering the rich experience in HAIC of our center, HAIC, as a pioneering treatment, was selected by our 
center after MDT in order to keep continuous hypertrophy of FLR and prevent tumor progression. The other main reasons are listed as follows: 1. HAIC exerts maximal antitumor effect by continuous high-concentration perfusion of cytotoxic drugs while having little effect on normal liver tissue. It is safer and less likely to impair liver function and induce systemic adverse effects (12). 2. If inappropriately performed, TACE may cause necrosis of huge HCC and even normal liver tissue, resulting in acute complications with life-threatening risks. 3. Acute hypoxia of the liver can be caused by TACE and may lead to revascularization and local recurrence of the tumor (13). 4. A new randomized controlled trial on HAIC has reported that HAIC shows better efficacy than TACE in patients with HCC larger than $7 \mathrm{~cm}$ (14). 5. HAIC has been developed in Asian countries for many years, and it seems that HCC yields a better objective response rate with HAIC among Asians (15). To sum up, HAIC was naturally selected by us after stage I ALPPS in this case. Once the requirements were met, stage II ALPPS was encouragingly carried out.

The key feature of our case was the first usage of HAIC worldwide after stage I ALPPS with insufficient hypertrophy of the FLR for a patient with HCC. It seems that we have gotten a good outcome. Similar cases have never been reported before; so, we want to share with readers a novel method for treating patients with a colossal HCC.

\section{CONCLUSIONS}

A new procedure, HAIC with FOLFOX4, could be an alternative treatment in case of insufficient hypertrophy of the FLR after the first step of ALPPS. It is expected to win more opportunities for curative liver resection for patients with a huge HCC. We try to name the procedure as ALPPS -HAIC. However, few studies are

\section{REFERENCES}

1. Park JW, Chen M, Colombo M, Roberts LR, Schwartz M, Chen PJ, et al. Global patterns of hepatocellular carcinoma management from diagnosis to death: the BRIDGE study. Liver Int. (2015) 35:2155-66. doi: 10.1111/liv. 12818

2. Alvarez FA, Ardiles V, Sanchez Claria R, Pekolj J, de Santibanes E. Associating liver partition and portal vein ligation for staged hepatectomy (ALPPS): tips and tricks. J Gastrointest Surg. (2013) 17:814-21. doi: 10.1007/s11605-012-2092-2

3. D’Haese JG, Neumann J, Weniger M, Pratschke S, Bjornsson B, Ardiles V, et al. Should ALPPS be used for liver resection in intermediate-stage HCC? Ann Surg Oncol. (2016) 23:1335-43. doi: 10.1245/s10434-015-5007-0

4. Kishi Y, Abdalla EK, Chun YS, Zorzi D, Madoff DC, Wallace MJ, et al. Three hundred and one consecutive extended right hepatectomies: evaluation of outcome based on systematic liver volumetry. Ann Surg. (2009) 250:5408. doi: 10.1097/SLA.0b013e3181b674df

5. Hartke J, Johnson M, Ghabril M. The diagnosis and treatment of hepatocellular carcinoma. Semin Diagn Pathol. (2017) 34:1539. doi: 10.1053/j.semdp.2016.12.011

6. Kim HJ, Kim CY, Hur YH, Koh YS, Kim JC, Cho CK, et al. Comparison of remnant to total functional liver volume ratio and remnant to standard liver volume ratio as a predictor of postoperative liver function after liver resection. Korean J Hepatobiliary Pancreat Surg. (2013) 17:14351. doi: 10.14701/kjhbps.2013.17.4.143 conducted in this area. More studies are needed to confirm the safety, feasibility, and efficacy of our method.

\section{DATA AVAILABILITY STATEMENT}

The original contributions presented in the study are included in the article/supplementary material, further inquiries can be directed to the corresponding author/s.

\section{ETHICS STATEMENT}

Written informed consent was obtained from the individual(s) for the publication of any potentially identifiable images or data included in this article.

\section{AUTHOR CONTRIBUTIONS}

JM and JW performed the surgery. WZ designed the study and wrote the original draft. AL revised the manuscript. WY and JD collected and arranged imaging and pathological data. All the authors contributed to the article and approved the submitted version.

\section{FUNDING}

The National Natural Science Foundation of China, and its grant numbers is 81702406 .

\section{ACKNOWLEDGMENTS}

We thank the imaging department and pathology department for technical support in the diagnosis of the patient and treatment process.
7. Shindoh J, Tzeng CWD, Vauthey JN. Portal vein embolization for hepatocellular carcinoma. Liver Cancer. (2012) 1:15967. doi: $10.1159 / 000343829$

8. van Lienden KP, van den Esschert JW, de Graaf W, Bipat S, Lameris JS, van Gulik TM, et al. Portal vein embolization before liver resection: a systematic review. Cardiovasc Intervent Radiol. (2013) 36:25-34. doi: 10.1007/s00270-012-0440-y

9. Romic B, Romic I, Mance M, Pavlek G, Skegro M. Successful associating liver partition and portal vein ligation after unsuccessful double TACE procedure complicated with sepsis and pancreatitis. Klin Onkol. (2016) 29:5962. doi: 10.14735 /amko201659

10. Chan A, Zhang WY, Chok K, Dai J, Ji R, Kwan C, et al. ALPPS versus portal vein embolization for hepatitis-related hepatocellular carcinoma: a changing paradigm in modulation of future liver remnant before major hepatectomy. Ann Surg. (2021) 273:957-65. doi: 10.1097/SLA.0000000000003433

11. Wang Z, Peng Y, Sun Q, Qu X, Tang M, Dai Y, et al. Salvage transhepatic arterial embolization after failed stage I ALPPS in a patient with a huge HCC with chronic liver disease: a case report. Int J Surg Case Rep. (2017) 39:131-5. doi: 10.1016/j.ijscr.2017.07.034

12. Obi S, Sato S, Kawai T. Current status of hepatic arterial infusion chemotherapy. Liver Cancer. (2015) 4:188-99. doi: 10.1159/000367746

13. Raoul JL, Forner A, Bolondi L, Cheung TT, Kloeckner R, de Baere T. Updated use of TACE for hepatocellular carcinoma treatment: how and when to use it based on clinical evidence. Cancer Treat Rev. (2019) 72:2836. doi: 10.1016/j.ctrv.2018.11.002 
14. Shi M, Li Q, He M, Guo R. Hepatic arterial infusion chemotherapy (HAIC) with oxaliplatin, fluorouracil, and leucovorin (FOLFOX) versus transarterial chemoembolization (TACE) for unresectable hepatocellular carcinoma (HCC): a randomised phase III trial. Ann Oncol. (2020) 31:S688. doi: 10.1016/j.annonc.2020.08.1097

15. Lyu N, Kong Y, Mu L, Lin Y, Li J, Liu Y, et al. Hepatic arterial infusion of oxaliplatin plus fluorouracil/leucovorin vs. sorafenib for advanced hepatocellular carcinoma. J Hepatol. (2018) 69:60-9. doi: 10.1016/j.jhep.2018.02.008

Conflict of Interest: The authors declare that the research was conducted in the absence of any commercial or financial relationships that could be construed as a potential conflict of interest.
Publisher's Note: All claims expressed in this article are solely those of the authors and do not necessarily represent those of their affiliated organizations, or those of the publisher, the editors and the reviewers. Any product that may be evaluated in this article, or claim that may be made by its manufacturer, is not guaranteed or endorsed by the publisher.

Copyright (c) 2021 Zhuo, Li, Yang, Duan, Min and Wei. This is an open-access article distributed under the terms of the Creative Commons Attribution License (CC BY). The use, distribution or reproduction in other forums is permitted, provided the original author(s) and the copyright owner(s) are credited and that the original publication in this journal is cited, in accordance with accepted academic practice. No use, distribution or reproduction is permitted which does not comply with these terms. 